GA-A23921

\title{
THE EFFECT OF TURBULENT STRESSES ON THE EXPERIMENTAL DETERMINATION OF A THERMAL DIFFUSIVITY DUE TO TURBULENT TRANSPORT
}

\author{
by \\ D.R. BAKER
}




\section{DISCLAIMER}

This report was prepared as an account of work sponsored by an agency of the United States Government. Neither the United States Government nor any agency thereof, nor any of their employees, makes any warranty, express or implied, or assumes any legal liability or responsibility for the accuracy, completeness, or usefulness of any information, apparatus, product, or process disclosed, or represents that its use would not infringe privately owned rights. Reference herein to any specific commercial product, process, or service by trade name, trademark, manufacturer, or otherwise, does not necessarily constitute or imply its endorsement, recommendation, or favoring by the United States Government or any agency thereof. The views and opinions of authors expressed herein do not necessarily state or reflect those of the United States Government or any agency thereof. 


\title{
THE EFFECT OF TURBULENT STRESSES ON THE EXPERIMENTAL DETERMINATION OF A THERMAL DIFFUSIVITY DUE TO TURBULENT TRANSPORT
}

\author{
by \\ D.R. BAKER
}

This is a preprint of a paper to be submitted to Plasmas.

Phys. and Controlled Fusion.

Work supported by the U.S. Department of Energy under Contract No. DE-AC03-99ER54463 


\begin{abstract}
In plasmas where the transport processes are dominated by turbulence, it is not always straightforward to identify the magnitude of the experimental transport diffusion coefficients. This is primarily due to the fact that with turbulent transport, the separation of the convective and conductive terms depends upon the type of turbulence and/or the model which is used to describe it. For the energy transport it is not just a matter of deciding whether the convection term is $5 / 2$ or $3 / 2$ times the product of the particle flux and the temperature. It is also important to identify turbulence generated convection terms such as heat pinches which can obscure the correct evaluation of the thermal diffusivity. Here we show that inclusion of the turbulence induced stresses into the transport model identifies a heat pinch term and changes the expression for the thermal diffusivity. Comparison with experimental results shows that the new calculated ion thermal diffusivity is no longer less than the neoclassical value even for plasmas with very good ion thermal confinement.
\end{abstract}




\section{INTRODUCTION}

As part of the effort to obtain a predictive understanding of transport in a magnetized plasma, analytical theoretical predictions and the results from numerical codes are compared with various measured quantities from experiments. A common comparison which is often made is between a theoretical and experimentally determined value for $\chi$, the thermal diffusivity. Specially, in the area of tokamak transport research, the theoretical $\chi$ is often chosen to be the "neoclassical" value, where "neoclassical" refers to the inclusion of orbit effects due to the tokamak toroidal geometry in addition to the collisions which are the basic cause of neoclassical transport. ${ }^{1-5}$ In this paper we will use the Hinton-Hazeltine version which was subsequently modified by Chang-Hinton. ${ }^{4} \mathrm{We}$ will refer to this as the Chang-Hinton neoclassical thermal diffusivity.

In the case of transport due to electrostatic or electromagnetic turbulence it is possible to develop a transport matrix, ${ }^{6-8}$ but difficulties arise due to the fact that each type of turbulence has different dependencies on the various gradients. From an experimental view it is difficult to identify the diffusive and convective terms since it is not always possible to identify the type of turbulence in any specific region of the plasma. As an example, a component of the particle flux that is commonly discussed is the term that is given by the ensemble and flux surface average of the product of the fluctuating density and the fluctuating $\mathrm{E} \times \mathrm{B}$ velocity, $\left\langle\tilde{n} \tilde{\mathbf{v}}_{\mathrm{E}}\right\rangle$. On the face of it, this part of the particle flux describes the convective transport of particle density and thus is a convective term. However if $\tilde{n}$ or $\tilde{\mathbf{v}}_{\mathrm{E}}$ depend directly upon the density gradient, it could be considered that this term has diffusive components.

The paper is organized as follows. In Section II, velocity moments are taken of the general kinetic equation which provide particle and energy balance equations and allow the development of expressions for the particle and energy flux. In Section III an expansion of the drift kinetic equation is used to obtain expressions for the fluxes and then a de facto closure of the moments equations is obtained by relating the fluxes derived by the two techniques. In Section IV, the expressions for diffusion coefficients for turbulent transport are discussed and compared to a specific set of analyzed DIII-D 9 data. A summary is presented in Section V. 


\section{MOMENT EQUATIONS}

The goal here is to produce expressions for the radial particle and ion energy fluxes which can be compared with experimental results. We will start from the following moments of the general kinetic equation ${ }^{10}$

$$
\frac{\partial n}{\partial t}+\nabla \cdot(n \overrightarrow{\boldsymbol{u}})=S_{\mathrm{p}}
$$

where $\quad n \equiv \int d^{3} \mathrm{v} f$ and $\overrightarrow{\boldsymbol{u}} \equiv \frac{1}{n} \int d^{3} \mathrm{v} f \overrightarrow{\mathbf{v}}$,

$$
m n\left[\frac{\partial \overrightarrow{\boldsymbol{u}}}{\partial t}+(\overrightarrow{\boldsymbol{u}} \cdot \nabla) \overrightarrow{\boldsymbol{u}}\right]+\nabla p-e n(\overrightarrow{\boldsymbol{E}}+\overrightarrow{\boldsymbol{u}} \times \overrightarrow{\boldsymbol{B}} / c)=\overrightarrow{\boldsymbol{S}}_{\mathrm{m}}
$$

where $\quad p \equiv \frac{m}{3} \int d^{3} \mathrm{v} f|\overrightarrow{\mathbf{v}}-\overrightarrow{\boldsymbol{u}}|^{2}$, is the scalar pressure,

$$
\frac{3}{2} \frac{\partial p}{\partial t}+\frac{1}{2} \frac{\partial n m u^{2}}{\partial t}+\nabla \cdot \overrightarrow{\boldsymbol{Q}}-e n \overrightarrow{\boldsymbol{u}} \cdot \overrightarrow{\boldsymbol{E}}=\overrightarrow{\boldsymbol{S}}_{\varepsilon},
$$

where $\quad \overrightarrow{\boldsymbol{Q}} \equiv \frac{m}{2} \int d^{3} \mathrm{v} f \mathrm{v}^{2} \overrightarrow{\mathbf{v}}$, is the total energy flux,

$$
\frac{\partial \overrightarrow{\boldsymbol{Q}}}{\partial t}+\nabla \cdot \overrightarrow{\boldsymbol{R}}-\frac{5}{2} \frac{e}{m} p \overrightarrow{\boldsymbol{E}}-\frac{1}{2} e n u^{2} \overrightarrow{\boldsymbol{E}}-\frac{e}{m c} \overrightarrow{\boldsymbol{Q}} \times \overrightarrow{\boldsymbol{B}}=0
$$

where $\quad \overleftrightarrow{\boldsymbol{R}} \equiv \frac{m}{3} \int d^{3} \mathrm{v} f \mathrm{v}^{2} \overrightarrow{\mathbf{v}} \overrightarrow{\mathbf{v}}$, is the energy weighted stress.

$\overrightarrow{\mathbf{v}}$ is the particle velocity. The distribution function, $f$, consists of a slowly varying part, $\bar{f}=\langle f\rangle$, and a fluctuating part $\tilde{f}$. Thus all quantities which are defined by velocity integrals of $f$ consist of a slowly varying part and a fluctuating part. $\overrightarrow{\boldsymbol{E}}$, the electric field is assumed to consist of a slowly varying part, in the toroidal and poloidal directions, and an electrostatic fluctuating part. $\overrightarrow{\boldsymbol{B}}$, the magnetic field is assumed to be constant in time. Since we are only interested in turbulent transport and not collisional transport, all explicit collisional terms are omitted. Important implicit collisional effects are included in the assumption of a scalar pressure and in the source terms. $S_{\mathrm{p}}$, the particle source, which includes neutral beam particles, particles entering the plasma at the edge and other neutral sources, requires collisional ionization processes. $\vec{S}_{\mathrm{m}}$, the momentum source, requires collisional viscosity acting on the injected beams. $S_{\varepsilon}$, the energy source, 
involves collisional slowing down of fast injected beam ions and the slowing of fast particles generated by rf. $S_{\varepsilon}$ also includes the collisional energy exchange between species. Although these equations apply to all species, here we will limit ourselves to consider only the ions and, for notational simplicity, to assume that there is only one ionic species.

Since we are interested in comparing with experimental measurements which are made by averaging over some time period that is larger than the fluctuation period, we will take a time average of the equations. We will assume that this time average is equivalent to an ensemble average. We will also assume that the plasma has reached a quasi stationary state and thus the partial derivatives with respect to time of the averaged quantities will be neglected. Following the usual technique $6,7,10,11$ a formal expression for the particle flux and the energy flux can be obtained by taking the cross product of Eqs. (2) and (4) with $\overrightarrow{\boldsymbol{B}}$ and then taking the radial component. In this work, radial $(\hat{\boldsymbol{r}})$ refers to a direction normal to the magnetic flux surfaces. The result for the particle flux is,

$$
\langle\Gamma\rangle_{\mathrm{r}} \equiv\langle n \overrightarrow{\boldsymbol{u}}\rangle \cdot \hat{\boldsymbol{r}}=\frac{1}{\Omega}\langle n\{\hat{\boldsymbol{b}} \times[(\overrightarrow{\boldsymbol{u}} \cdot \nabla) \overrightarrow{\boldsymbol{u}}]\}\rangle \hat{\boldsymbol{r}}+\left\langle\tilde{n} \tilde{\mathbf{v}}_{E}\right\rangle \cdot \hat{\boldsymbol{r}}=\left\langle\Gamma_{\mathrm{S}}\right\rangle_{\mathrm{r}}+\left\langle\Gamma_{\mathrm{E}}\right\rangle_{\mathrm{r}}
$$

where $\hat{\boldsymbol{b}} \equiv \overrightarrow{\boldsymbol{B}} / B$, with $B$ the magnitude of the magnetic field, $\Omega$ is the ion cyclotron frequency and $\tilde{\mathbf{v}}_{\mathrm{E}} \equiv c(\boldsymbol{E} \times \boldsymbol{b}) / B$, with $c$ the speed of light. In this section the angle brackets refer to a combined ensemble average and flux surface average. Later in the paper we will distinguish between ensemble, flux and orbit averages. The $\mathrm{r}$ subscript means in the $\hat{\boldsymbol{r}}$ direction. Equation (5) is essentially Eq. (4) from Reference 6, but without the collisional and electromagnetic terms.

The particle flux consists of two terms, the well known $\left\langle\tilde{n} \tilde{\mathbf{v}}_{\mathrm{E}}\right\rangle$ term and a term which is related to the turbulence generated Reynolds stress. It is instructive to estimate the relative size of these two terms. It is difficult to estimate the size of $\left\langle\tilde{n} \tilde{\mathbf{v}}_{\mathrm{E}}\right\rangle$ since it depends on the phase difference between $\tilde{\boldsymbol{n}}$ and $\tilde{\mathbf{v}}_{\mathrm{E}}$, which to first or second order is 90 degrees. However, it is safe to assume that $\left\langle\Gamma_{\mathrm{E}}\right\rangle_{\mathrm{r}}=\left\langle\tilde{n} \tilde{\mathbf{v}}_{\mathrm{E}}\right\rangle \cdot \hat{\boldsymbol{r}} \leq\langle\Gamma\rangle_{\mathrm{r}} \equiv \bar{n} \bar{u}_{\mathrm{r}}$ (where is a short hand notation for \langle\rangle ). In other words, the total radial particle flux, given by $\bar{n} \bar{u}_{\mathrm{r}}^{*}$, is an upper bound on $\left\langle\Gamma_{\mathrm{E}}\right\rangle_{\mathrm{r}}$. The flux due to the stresses, $\left\langle\Gamma_{\mathrm{S}}\right\rangle_{\mathrm{r}}$, can be estimated from the approximate size of one of its largest components,

$$
\left\langle\vec{\Gamma}_{\mathrm{S}}\right\rangle_{\mathrm{r}} \sim \frac{\bar{n}}{\Omega}\left\{\left|\tilde{u}_{\mathrm{r}}\right| \frac{\partial\left|\tilde{u}_{\theta}\right|}{\partial r}\right\} \sim \bar{n} \bar{u}_{\mathrm{r}}^{*} k_{\perp} \rho_{\mathrm{L}} \frac{\left|\tilde{u}_{\mathrm{r}}\right|\left|\tilde{u}_{\theta}\right|}{\bar{u}_{\mathrm{r}}^{*} \mathrm{v}_{\text {thi }}},
$$

where $k_{\perp}$ is the average perpendicular wave number, $\rho_{\mathrm{L}}$ is the ion Larmor radius and $\mathrm{v}_{\text {thi }}$ is the ion thermal velocity. The ensemble average of the product $\left\langle\tilde{u}_{\mathrm{r}}\left(\partial \tilde{u}_{\theta} / \partial r\right)\right\rangle$ can 
be approximately replaced by the product of the magnitudes since $\tilde{u}_{\mathrm{r}}$ and $\partial \tilde{u}_{\theta} / \partial r$ are approximately in phase. ${ }^{12}$ It is well known that $\tilde{\mathrm{u}} \approx \tilde{\mathbf{v}}_{E} \cdot{ }^{13} \mathrm{We}$ then assume that,

$$
\tilde{u}_{\mathrm{r}} \sim \tilde{u}_{\theta} \sim c k_{\perp} \tilde{\phi} / B \sim\left(k_{\perp} \rho_{\mathrm{L}}\right)(e \tilde{\phi} / T) \mathrm{v}_{\text {thi }}
$$

where $\tilde{\phi}$ is the fluctuating electric potential and $T$ is the ion temperature. Then,

$$
\left\langle\vec{\Gamma}_{\mathrm{S}}\right\rangle_{\mathrm{r}} \sim \bar{n} \bar{u}_{\mathrm{r}}^{*}\left(k_{\perp} \rho_{\mathrm{L}}\right)^{3}\left(\frac{e \tilde{\phi}}{T}\right)^{2} \frac{\bar{u}_{\mathrm{r}}^{*}}{\mathrm{v}_{\mathrm{thi}}}
$$

Experimental measurement and simulations 14,15 show that $0.2 \leq k_{\perp} \rho_{\mathrm{L}} \leq 0.6$, and measurement 14,16 shows that, depending upon position in the plasma, $10^{-3} \leq(e \tilde{\phi} / T) \leq 0.15$. In most DIII-D L-mode discharges, it is found that, depending upon position in the plasma, $10^{7} \geq \mathrm{v}_{\text {thi }} / \bar{u}_{\mathrm{r}}^{*} \geq 10^{5}$. Typical values for $(e \bar{\phi} / T)^{\sim}\left(\mathrm{v}_{\text {thi }} / \bar{u}_{\mathrm{r}}^{*}\right)$ range from 2-200, and thus for many L-mode plasmas there is a large region where $\left\langle\vec{\Gamma}_{S}\right\rangle_{\mathrm{r}} \sim\langle\Gamma\rangle_{\mathrm{r}} \geq\left\langle\Gamma_{E}\right\rangle_{\mathrm{r}}$. Thus $\left\langle\vec{\Gamma}_{S}\right\rangle_{\mathrm{r}}$ cannot be neglected and may be the largest component of the particle flux, depending on the strength of the turbulence and other plasma parameters.

The energy flux is also found to have two terms,

$$
\langle\mathrm{Q}\rangle_{r}=\frac{1}{\Omega}\langle\hat{\mathrm{b}} \times \nabla \cdot \overrightarrow{\boldsymbol{R}}\rangle \cdot \hat{\mathrm{r}}+\frac{5}{2}\left\langle\tilde{\mathrm{p}} \tilde{\mathbf{v}}_{\mathrm{E}}\right\rangle \cdot \hat{\mathrm{r}}=\left\langle\mathrm{Q}_{\mathrm{S}}\right\rangle_{r}+\left\langle\mathrm{Q}_{\mathrm{E}}\right\rangle_{r}
$$

We have made no attempt to close the moment equations and it is not possible to obtain an expression for $\overrightarrow{\boldsymbol{R}}$ from Eqs. (1) through (4), but for purposes of size estimation, we postulate that from the definition of $\overrightarrow{\boldsymbol{R}}$ in Eq. (4), $\left\langle Q_{S}\right\rangle_{\mathrm{r}}$ will have terms like $(5 / 2)(1 / \Omega)\langle n T\{\boldsymbol{b} \times\lfloor(\overrightarrow{\boldsymbol{u}} \cdot \nabla) \overrightarrow{\boldsymbol{u}}]\}\rangle \cdot \hat{\boldsymbol{r}}$. Therefore, using similar estimations as for the particle flux, we see that $\left\langle Q_{S}\right\rangle_{\mathrm{r}}$ cannot be neglected with respect to $\left\langle Q_{\mathrm{E}}\right\rangle_{\mathrm{r}}$.

For notational brevity, Eq. (2) utilized the usual "tokamak approximation"10 that the pressure is a scalar. However since the inertial term, in the form of $(\overrightarrow{\boldsymbol{u}} \cdot \nabla) \overrightarrow{\boldsymbol{u}}$, is included, the non diagonal part of the pressure tensor should also be considered. These terms can be included in $\left\langle\Gamma_{S}\right\rangle_{\mathrm{r}}$ by changing its definition. This does not change the size estimation of Eq. (6).

In the next section expressions for $\left\langle\vec{\Gamma}_{S}\right\rangle_{\mathrm{r}}$ and $\left\langle Q_{S}\right\rangle_{\mathrm{r}}$ will be developed using the drift kinetic equation. 


\section{DRIFT KINETIC EQUATION}

The drift kinetic equation for the ions can be written as $10,17,18$

$$
\frac{\partial \breve{\mathrm{f}}}{\partial \mathrm{t}}+\left(\overrightarrow{\mathbf{v}}_{\|}+\overrightarrow{\mathbf{v}}_{d}\right) \cdot \nabla \breve{\mathrm{f}}+\mathrm{e}\left(\overrightarrow{\mathbf{v}}_{\|}+\overrightarrow{\mathbf{v}}_{d}\right) \cdot \overrightarrow{\boldsymbol{E}} \frac{\partial \breve{\mathrm{f}}}{\partial \mathrm{K}}=\mathrm{C}(\breve{\mathrm{f}})
$$

where $\breve{f}$ is a distribution function which is averaged over the rapidly varying gyrophase of the particles. $\overrightarrow{\mathbf{v}}_{\|}$is the guiding center velocity along the magnetic field line and $\overrightarrow{\mathbf{v}}_{d}$ is the guiding center drift velocity. $\overrightarrow{\boldsymbol{E}}$ is the total electric field, $K$ is the particle kinetic energy and $C$ is a collision operator. In order to complete the particle description, source terms are also needed, but are usually omitted for notational brevity. As in the previous section, the turbulence is assumed to be electrostatic, with the time derivative of the magnetic field, $\partial B / \partial t$, equal to zero. Also in the following, only the equation for the ions will be considered and impurities will be ignored.

Following Reference 8, the distribution function and the drift velocity are expressed in terms of an ensemble averaged part and a fluctuating part,

$$
\begin{aligned}
& \breve{f}=\langle\breve{f}\rangle_{\mathrm{ens}}+\tilde{f}, \\
& \overrightarrow{\mathbf{v}}_{\mathrm{d}}=\left\langle\overrightarrow{\mathbf{v}}_{\mathrm{d}}\right\rangle_{\mathrm{ens}}+\overrightarrow{\mathbf{v}}_{\mathrm{d}} \equiv \overrightarrow{\mathbf{v}}_{\mathrm{D}}+\tilde{\mathbf{v}}_{\mathrm{E}} \equiv \overrightarrow{\mathbf{v}}_{\mathrm{d} 0}+\overrightarrow{\mathbf{v}}_{\mathrm{E}} \equiv \overrightarrow{\mathbf{v}}_{\mathrm{d} 0}+\overrightarrow{\mathbf{v}}_{\mathrm{E} 0}+\tilde{\mathbf{v}}_{\mathrm{E}}, \\
& \overrightarrow{\mathbf{v}}_{\mathrm{d} 0}=\frac{\hat{\boldsymbol{b}}}{\Omega} \times\left[\mathrm{v}_{\|}^{2}+\frac{\mathrm{v}_{\perp}^{2}}{2}\right] \frac{\nabla B}{B}, \quad \overrightarrow{\mathbf{v}}_{\mathrm{E}}=\frac{c \overrightarrow{\boldsymbol{E}} \times \hat{b}}{B}, \overrightarrow{\boldsymbol{E}}=-\nabla \phi_{0}-\nabla \tilde{\phi},
\end{aligned}
$$

where $\phi$ is the electric potential.

Two ordering parameters are specified. $\Delta$ is the fluctuation amplitude expansion parameter, with,

$$
\frac{\tilde{f}}{\bar{f}} \sim \frac{f_{1}}{f_{\mathrm{M}}} \sim \frac{e \tilde{\phi}}{T} \sim \frac{\tilde{n}}{\bar{n}} \leq \frac{a}{q R} \sim \Delta<<1,
$$

and $\delta$ is the drift ordering parameter given by

$$
\delta \sim \rho_{\mathrm{L}} / a
$$


where $\rho_{\mathrm{L}}$ is the average Larmor radius of the ions, $R$ is the major radius and $a$ is the minor radius of the tokamak. Here, it is assumed that $k_{\|} \approx 1 / q R$, where $k_{\|}$is the wave number parallel to the magnetic field. Experimental measurements show that $0.2 \leq k_{\perp} \rho_{\mathrm{L}} \leq 0.6 .15$ Since $a / q R \sim \Delta$ and $\delta \sim \Delta^{2}$, this implies that $k_{\|} / k_{\perp} \sim \delta$. The distribution function is expanded to order $\Delta, 8$

$$
\breve{f}=\left\langle\breve{f}_{0}\right\rangle_{\mathrm{ens}}+\left\langle\breve{f}_{1}\right\rangle_{\mathrm{ens}}+\tilde{f}+\vartheta(\delta) \equiv f_{\mathrm{M}}+f_{1}+\tilde{f}+\vartheta(\delta)
$$

From the definitions for $n, \overrightarrow{\boldsymbol{u}}$ and $\overrightarrow{\boldsymbol{Q}}$ in Eqs. (1) and (3), we can write expressions for the particle flux and the energy flux in terms of the drift velocities,

$$
\begin{aligned}
& \langle\Gamma\rangle_{\mathrm{r}} \equiv \bar{\Gamma}=\sigma_{\psi}\left\langle\int d^{3} \mathrm{v}\left\langle\overrightarrow{\mathbf{v}}_{\mathrm{d}} \cdot \nabla \psi \breve{f}\right\rangle_{\mathrm{ens}}\right\rangle_{\mathrm{fs}} / A, \\
& \langle Q\rangle_{\mathrm{r}} \equiv \bar{Q}=\sigma_{\psi}\left\langle\int d^{3} \mathrm{v} K\left\langle\overrightarrow{\mathbf{v}}_{\mathrm{d}} \cdot \nabla \psi \breve{f}\right\rangle_{\mathrm{ens}}\right\rangle_{\mathrm{fs}} / A,
\end{aligned}
$$

where, for the remainder of the paper, \langle\rangle$_{\text {ens }}$ denotes the ensemble average, \langle\rangle$_{\text {fs }}$ denotes the non-normalized flux surface average and $A$ is the flux surface area. $\sigma_{\psi}$ is the sign of $\nabla \psi$. For notational simplicity, it will be assumed here that both the toroidal and poloidal magnetic fields are positive and thus $\sigma_{\psi}$ is positive and will be dropped. The magnitude and radial direction of the transport is independent of the direction of the magnetic field.

Using Eqs. (12) and (15), we obtain

$$
\begin{gathered}
\bar{\Gamma}=\bar{\Gamma}_{\mathrm{a}}+\bar{\Gamma}_{\mathrm{b}}=\left\langle\int d^{3} \mathrm{v} \overrightarrow{\mathbf{v}}_{\mathrm{d} 0} \cdot \nabla \psi f_{1}\right\rangle_{\mathrm{fs}} / A+\left\langle\int d^{3} \mathrm{v}\left\langle\tilde{\mathrm{v}}_{\mathrm{E}} \cdot \nabla \psi \tilde{f}\right\rangle_{\mathrm{ens}}\right\rangle_{\mathrm{fs}} / A \\
\bar{Q}=\bar{Q}_{\mathrm{a}}+\bar{Q}_{\mathrm{b}}=\left\langle\int d^{3} \mathrm{v} K \overrightarrow{\mathbf{v}}_{\mathrm{d} 0} \cdot \nabla \psi f_{1}\right\rangle_{\mathrm{fs}} / A+\left\langle\int d^{3} \mathrm{v} K\left\langle\tilde{\mathrm{v}}_{\mathrm{E}} \cdot \nabla \psi \tilde{f}\right\rangle_{\mathrm{ens}}\right\rangle_{\mathrm{fs}} / A .
\end{gathered}
$$

Comparison of Eqs. (18) and (19) with (5) and (8), it is clear that $\bar{Q}_{\mathrm{b}}=\left\langle Q_{\mathrm{E}}\right\rangle_{\mathrm{r}}$ and $\bar{\Gamma}_{\mathrm{b}}=$ $\left\langle\Gamma_{\mathrm{E}}\right\rangle_{\mathrm{r}}$ and thus we can infer that $Q_{\mathrm{a}} \approx\left\langle Q_{S}\right\rangle_{\mathrm{r}}$ and $\Gamma_{\mathrm{a}} \approx\left\langle\Gamma_{S}\right\rangle_{\mathrm{r}}$. An integration by parts shows that $\bar{\Gamma}_{\mathrm{a}}$ derives from the $\overrightarrow{\mathbf{v}} \cdot \nabla \mathrm{f}$ term of the kinetic equation. This is also true for $\left\langle\Gamma_{S}\right\rangle_{\mathrm{r}}$.

An approximate expression for $\bar{\Gamma}_{\mathrm{a}}$ has been obtained elsewhere ${ }^{19}$ and is given by,

$$
\bar{\Gamma}_{\mathrm{a}} \approx-D \frac{\partial \bar{n}}{\partial \rho}+V_{\mathrm{p}} \bar{n}
$$


with

$$
D=2 \pi I^{2} \frac{m c^{2}}{T} \frac{R_{0}}{B_{\mathrm{T} 0}^{2}} \frac{q^{2} \mathcal{H}}{\rho}\left\langle\frac{\left|B_{\theta}\right|}{B^{2}}\left\langle k_{\theta} \Delta \omega_{*}|\tilde{\phi}|^{2}\right\rangle_{\mathrm{ens}}\right\rangle
$$

and

$$
V_{\mathrm{p}}=D q \mathcal{H} \frac{\partial(q \mathcal{H})^{-1}}{\partial \rho}
$$

where, $m$ is the ion mass, $R_{0}$ is the major radius at the magnetic axis, $B_{\mathrm{T} 0}$ is the toroidal magnetic field at the magnetic axis, $q$ is the usual tokamak safety factor. The geometric factor $\mathcal{H}(\rho)$ is 1.0 for centered circles and ranges from near 1.0, at the center, to near 0.7, at the edge, for most $\mathrm{D}$ shaped non-circular cross section plasmas. $\rho$ is the average radius defined by $\rho^{2} \equiv \pi B_{\mathrm{T} 0} \Phi$, with $\Phi$ the toroidal flux. $B_{\theta}$ is the poloidal magnetic field and

$$
\Delta \omega_{*} \equiv\left|\sum_{\mathrm{k}}\left[\omega_{\mathrm{d}}(k)-\omega_{*}(k)\right]\right|
$$

with $\omega_{d}=\overrightarrow{\boldsymbol{k}} \cdot \overrightarrow{\mathbf{v}}_{d}$ and

$$
\omega_{*}=k_{\theta} \frac{c T}{e B} \frac{1}{L_{\mathrm{n}}}\left[1+\left(\frac{K}{T}-\frac{3}{2}\right) \eta_{\mathrm{i}}\right]+k_{\theta} \mathrm{v}_{\mathrm{E} 0}
$$

The energy flux can be obtained in a similar way and is given by,

$$
\bar{Q}_{\mathrm{a}} \approx-\frac{3}{2} D \frac{\partial \bar{n} \bar{T}}{\partial \rho}+\frac{3}{2} V_{\mathrm{p}} \bar{n} \bar{T},
$$

which can be written as,

$$
\overline{\mathrm{Q}}_{a} \approx-\chi_{i, a} \overline{\mathrm{n}} \frac{\partial \overline{\mathrm{T}}}{\partial \rho}+\frac{3}{2} \bar{\Gamma}_{a} \overline{\mathrm{T}},
$$

where we have defined $\chi_{i, a} \equiv(3 / 2 D)$.In the next section, the results of Eqs. (18) through (22) are used with the particle and energy conservation equations, Eqs (1) and (3), in order to obtain approximate relations for the effective thermal and particle diffusion coefficients. 


\section{EXPRESSIONS FOR $\chi_{i}$ AND D}

In steady state, the relation between the particle flux and the (presumed known) sources is given by,

$$
\bar{\Gamma} \approx-D \frac{\partial \bar{n}}{\partial \rho}+V_{\mathrm{p}} \bar{n}+\left\langle\tilde{n} \tilde{\mathbf{v}}_{\mathrm{E}}\right\rangle=\frac{1}{V^{\prime}} \int d V S_{\mathrm{p}},
$$

where $V$ is the local flux surface volume and $V^{\prime}$ is $\partial V / \partial \rho$. Expressions for $\left\langle\tilde{n} \tilde{\mathbf{v}}_{E}\right\rangle$ have been derived elsewhere. ${ }^{6-8}$ This part of the particle flux comes from the particles which interact resonantly with the turbulence. It is proportional to $|e \tilde{\phi} / T|^{<}$as are $D$ and $V_{\mathrm{p}}$. The dependence of this term on the density gradient depends upon the type of unstable turbulence which is present in the plasma and it is not clear whether to include it into the diffusive term or the convective term, however since it has the mathematical form of a convection of particle density, perhaps it is more appropriate to think of it as a convective term. Nonetheless, it is common to combine all of the terms in Eq. (23) into one diffusive type term, $\Gamma \equiv-D_{\text {eff }} \partial \bar{n} / \partial \rho$.

The experimental evidence from DIII-D low confinement mode (L-mode) discharges shows that the density profiles tend to be proportional to $(1 / q \mathcal{H})^{0.8} .{ }^{21}$ From Eq. (20) we see that one possible interpretation of this result is that the $\left\langle\tilde{\mathbf{n}} \tilde{\mathbf{v}}_{E}\right\rangle$ term is small and the small total flux is a balance between $-D \partial \bar{n} / \partial \rho$ and $V_{\mathrm{p}} \bar{n}$. Unfortunately this does not help much in getting an accurate value for the physical diffusion coefficient, since $D$ (a "large" value) is given by

$$
-\bar{\Gamma} /\left[\frac{\partial \bar{n}}{\partial \rho}-\left(\frac{V_{\mathrm{p}}}{\mathrm{D}}\right)\right],
$$

a "small" measured value divided by an even "smaller" measured value, all of which have moderately large measurement errors. 20

The experimental value for $\chi_{i}$ is obtained from the energy conservation equation, Eq. (3). The $\overrightarrow{\boldsymbol{u}} \cdot \overrightarrow{\boldsymbol{E}}$ term can be eliminated by taking the dot product of Eq. (2) with $\overrightarrow{\boldsymbol{u}}$ and expressing $\overrightarrow{\boldsymbol{u}} \cdot \overrightarrow{\boldsymbol{E}}$ in terms of the pressure gradient and momentum source. Using the tokamak symmetries, in steady state we have, 


$$
\bar{Q}=-\chi_{i, a} \overline{\mathrm{n}} \frac{\partial \overline{\mathrm{T}}}{\partial \rho}+\frac{3}{2} \Gamma_{a} \overline{\mathrm{T}}+\frac{5}{2}\left\langle\tilde{\mathrm{p}}_{\mathrm{E}}\right\rangle=\frac{1}{V^{\prime}} \int d V\left(S_{\varepsilon \mathrm{m}}+\langle\overrightarrow{\boldsymbol{u}} \cdot \nabla p\rangle\right)
$$

where $S_{\varepsilon \mathrm{m}} \equiv S_{\varepsilon}-\overrightarrow{\boldsymbol{u}} \cdot \vec{S}_{\mathrm{m}}$. We have neglected terms on the order of $|u|^{2} / \mathrm{v}_{\text {thi }}^{2}$, except for $\overrightarrow{\boldsymbol{u}} \cdot \overrightarrow{\boldsymbol{S}}_{\mathrm{m}}$ which is usually retained. It is tempting to approximate the compression term, $\langle\overrightarrow{\boldsymbol{u}} \cdot \nabla p\rangle$, by $\bar{u}_{\mathrm{r}}^{*} \nabla \bar{p}$, however this is usually not a good approximation. Both $\overrightarrow{\boldsymbol{u}}$ and $\nabla p$ are fluctuating quantities and have large components in the poloidal as well as the radial direction. We can express $\langle\overrightarrow{\boldsymbol{u}} \cdot \nabla p\rangle$ by

$$
\langle\overrightarrow{\boldsymbol{u}} \cdot \nabla p\rangle=\nabla \cdot\langle p \overrightarrow{\boldsymbol{u}}\rangle-\langle p \nabla \cdot \overrightarrow{\boldsymbol{u}}\rangle \approx \nabla \cdot(\vec{\Gamma} \bar{T}+\bar{n}\langle T \overrightarrow{\boldsymbol{u}}\rangle)-\langle p \nabla \cdot \overrightarrow{\boldsymbol{u}}\rangle
$$

We can then write Eq. (24) as,

$$
-\chi_{i, a} \overline{\mathrm{n}} \frac{\partial \overline{\mathrm{T}}}{\partial \rho}+\frac{3}{2} \bar{\Gamma}_{a} \overline{\mathrm{T}}+\frac{5}{2}\left\langle\tilde{\mathrm{p}} \tilde{\mathbf{v}}_{\mathrm{E}}\right\rangle_{\mathrm{r}}-\bar{\Gamma} \overline{\mathrm{T}}-\overline{\mathrm{n}}\langle\tilde{T} \tilde{\boldsymbol{u}}\rangle_{\mathrm{r}}+\frac{1}{V^{\prime}} \int d V(\langle p \nabla \cdot \overrightarrow{\boldsymbol{u}}\rangle)=\frac{1}{V^{\prime}} \int d V S_{\varepsilon \mathrm{m}},
$$

where we have made the assumption that $\tilde{\boldsymbol{u}} \cdot \hat{\boldsymbol{r}}>>\overline{\boldsymbol{u}} \cdot \hat{\boldsymbol{r}}$. Inspection of Eqs. (25) and (26) shows that for turbulent transport, the compression term, when brought to the left hand side of the equation acts like an effective heat pinch, with a value of approximately $\overline{\Gamma \mathrm{T}}$.

In steady state, the momentum balance equation, Eq. (2) or (5), yields $\overrightarrow{\boldsymbol{u}}=\{\boldsymbol{b} \times[(\overrightarrow{\boldsymbol{u}} \cdot \nabla) \overrightarrow{\boldsymbol{u}}]\} / \Omega+\tilde{\mathbf{v}}_{\mathrm{E}}$, where the first term is much smaller than the second term. We use the approximation, $\overrightarrow{\boldsymbol{u}} \equiv \overrightarrow{\boldsymbol{u}}_{\mathrm{S}}+\tilde{\mathbf{v}}_{\mathrm{E}} \approx \tilde{\mathbf{v}}_{\mathrm{E}}$ and the fact that $\nabla \cdot \tilde{\mathbf{v}}_{\mathrm{E}}=0$ and the approximation that the combination, $\bar{n}\left\langle\tilde{T}_{\mathrm{S}}\right\rangle-\left\langle\int d V \mathrm{p} \nabla \cdot \overrightarrow{\boldsymbol{u}}_{\mathrm{S}}\right\rangle / V$, two small terms which tend to cancel, is small compared to the other individual terms. We then express,

$$
\frac{5}{2}\left\langle\tilde{p} \tilde{\mathbf{v}}_{\mathrm{E}}\right\rangle_{\mathrm{r}} \approx \frac{5}{2} \overline{\mathrm{n}}\left\langle\tilde{T}_{\mathbf{\mathbf { v }}}\right\rangle_{\mathrm{r}}+\frac{5}{2} \overline{\mathrm{T}}\left\langle\tilde{n} \tilde{\mathbf{v}}_{\mathrm{E}}\right\rangle_{\mathrm{r}}
$$

and obtain,

$$
-\chi_{i, a} \overline{\mathrm{n}} \frac{\partial \overline{\mathrm{T}}}{\partial \rho}+\frac{1}{2} \bar{\Gamma}_{a} \overline{\mathrm{T}}+\frac{3}{2} \bar{\Gamma}_{\mathrm{E}} \overline{\mathrm{T}}+\frac{3}{2} \overline{\mathrm{n}}\left\langle\tilde{T}_{\mathbf{v}_{\mathrm{E}}}\right\rangle_{\mathrm{r}}=\frac{1}{V^{\prime}} \int d V S_{\varepsilon \mathrm{m}}
$$

The first term of Eq. (27) is a diffusive or conductive term which comes from the turbulence induced stresses. The second term is the net convection term from the turbulence induced stresses and is $3 / 2 \bar{\Gamma}_{a} \overline{\mathrm{T}}$ minus the corresponding part of the compression term, $\bar{\Gamma}_{a} \overline{\mathrm{T}}$. The third term is the convection term due the $\mathrm{E} \times \mathrm{B}$ type flux, $5 / 2 \bar{\Gamma}_{E} \overline{\mathrm{T}}$ minus the corresponding part of the compression term, $\bar{\Gamma}_{E} \overline{\mathrm{T}}$. The fourth term is the remainder of the $\mathrm{E} \times \mathrm{B}$ type flux, $5 / 2 \bar{n}\left\langle T \tilde{\mathbf{v}}_{\mathrm{E}}\right\rangle_{\mathrm{r}}$ minus the corresponding part of the compression term, $\bar{n}\left\langle T \tilde{\mathbf{v}}_{\mathrm{E}}\right\rangle_{\mathrm{r}}$. The term on the right hand side of the equation is the net 
energy input to the ions, including the auxiliary heating and the electron-ion exchange term.

Equation (27) is then used to obtain an approximate value for a thermal diffusivity from the (presumed known) sources. The simplest thing to do is to define an effective thermal diffusivity by,

$$
\chi_{\text {i eff }} \equiv-\frac{q_{\text {total }}}{\bar{n} \partial \bar{T} / \partial \rho}
$$

with

$$
q_{\mathrm{total}} \equiv \frac{1}{V^{\prime}} \int d V S_{\varepsilon \mathrm{m}}
$$

It is often felt desirable to separate out the 'convective' term from the total energy flux in order to isolate the "conductive" term (and incidentally obtain a lower experimental value for $\chi_{i}$, when $\bar{\Gamma}$ is positive). This is done by subtracting $\alpha \bar{\Gamma} \bar{T}$ from both sides of Eq. (27), where $\alpha$ is a number, usually either $5 / 2$ or $3 / 2.11,21-22$ A value of $5 / 2$ is strictly only appropriate for collisional transport if $\langle\overrightarrow{\boldsymbol{u}} \cdot \nabla p\rangle$ is small or when $\langle\overrightarrow{\boldsymbol{u}} \cdot \nabla \bar{p}\rangle$ can be approximated by $\bar{u}_{\mathrm{r}}^{*} \nabla \bar{p}$. Inspection of Eq. (27) shows that it is not appropriate for turbulent transport. If $\chi_{\mathrm{i}, \mathrm{a}}$, and $\Gamma_{a}$ are small, then using $3 / 2$ would result in a "conductive" term which is approximately $3 / 2 \bar{n}\left\langle T \tilde{\mathbf{v}}_{\mathrm{E}}\right\rangle_{\mathrm{r}}$, however this is in fact a "convective" term, leading perhaps to the conclusion that Eq. (28) should be used rather than Eq. (27).

Experimentally, on DIII-D, it is often found that for good confinement discharges with an internal transport barrier (ITB), ${ }^{24}$ the use of $\alpha=5 / 2$ or $\alpha=3 / 2$ results in negative "conductive" terms and negative $\chi_{\mathrm{i}}$ 's. This can be understood if, in fact, $\chi_{\mathrm{i}, \mathrm{a}}$ and $\bar{\Gamma}_{\mathrm{a}}$ are large and $\bar{\Gamma}_{\mathrm{E}}$ and $\overline{\mathrm{n}}\left\langle\mathbf{I} \mathbf{v}_{E}\right\rangle_{r}$ are small. Then, from Eq. (27), we see that an $\alpha$ $=1 / 2$ is perhaps more appropriate and that, under these conditions, $\chi_{i} \approx \chi_{i, a}=3 / 2 \mathrm{D}$.

From a thermodynamic viewpoint, arguments, ${ }^{7,8,12,25}$ can be made that the convective component is either $5 / 2 \Gamma \mathrm{T}$ or $3 / 2 \Gamma \mathrm{T}$. Here it is necessary to use $\alpha=1 / 2$ to eliminate an effective anomalous heat pinch equal to $\Gamma \mathrm{T}$.

As an example we consider the results of a TRANSP 26 analysis of a DIII-D long pulse high performance quiescent double barrier (QDB) ${ }^{27}$ discharge. Figure 1 shows the time history of the relevant parameters of this discharge. There is a long period with an ITB when the density and temperatures are changing only slowly. This time period is depicted in the figure. We will consider the TRANSP results from this time period. 
Figure 2 shows a radial plot of $\chi_{i}$ from this discharge which has been previously published. 27 The time at which this $\chi_{i}$ was calculated is shown in Fig. 1. Although the plot is semi-log, it is clear that for $\rho<0.35, \chi_{i}$ (which is obtained here by using $\alpha=3 / 2$ )

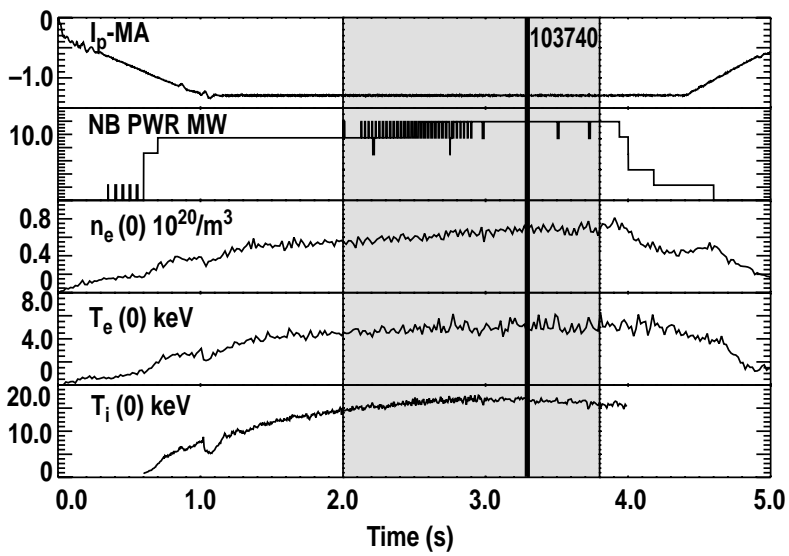

Fig. 1. Time histories of the plasma current, injected neutral beam power, central electron density, central electron temperature and central ion temperature for a DIII-D QDB type shot. The time range of interest is in the lightly shaded region. There is an internal transport barrier and an edge barrier during this time period. The dark line shows the time for the $\chi_{1}$ shown in Fig. 2.

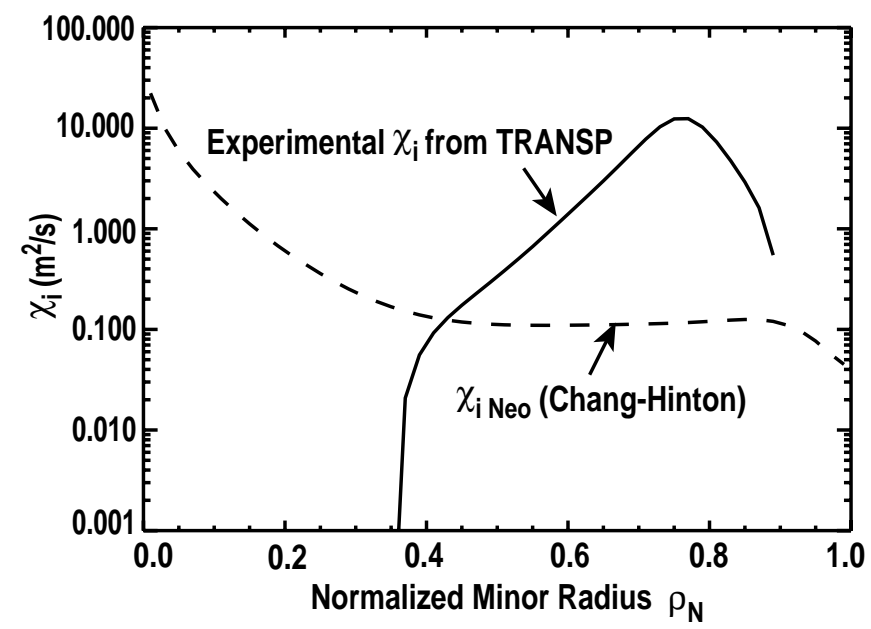

Fig. 2. Radial plot of $\chi_{1}$ at time 3.3 seconds in the discharge shown in Fig. 1. Also plotted is the Chang-Hinton value of the neoclassical $\chi_{i, n e o}$. For this result $\chi_{1}$ is calculated by using a "conductive" heat flux obtained by subtracting $3 / 2 \Gamma T$ from the total energy flux.

is negative. Also plotted for reference is the appropriate value of the Chang-Hinton neoclassical ion thermal diffusion coefficient, $\chi_{i, n e o}$. The calculation of $\chi_{i}$ tends to have large error since it requires the knowledge of the gradient of $T_{i}$. The ion temperature has errors in the measurement and is measured only at discrete spatial points, 
thus the gradient has large errors. The values of $q_{\text {cond }}$ from which $\chi_{i}$ is derived has smaller error. Figure 3 shows a radial plot of the $q_{\text {cond }}$, along with appropriate error bars, from which the $\chi_{i}$ in Fig. 2 was derived. For $\rho<0.35, q_{\text {cond }}$ is negative within the error bars. For comparison $q_{\text {total }}$ and $q_{\text {conv }} \equiv(3 / 2) \Gamma_{\mathrm{i}} T_{\mathrm{i}}$ are also plotted in this figure.

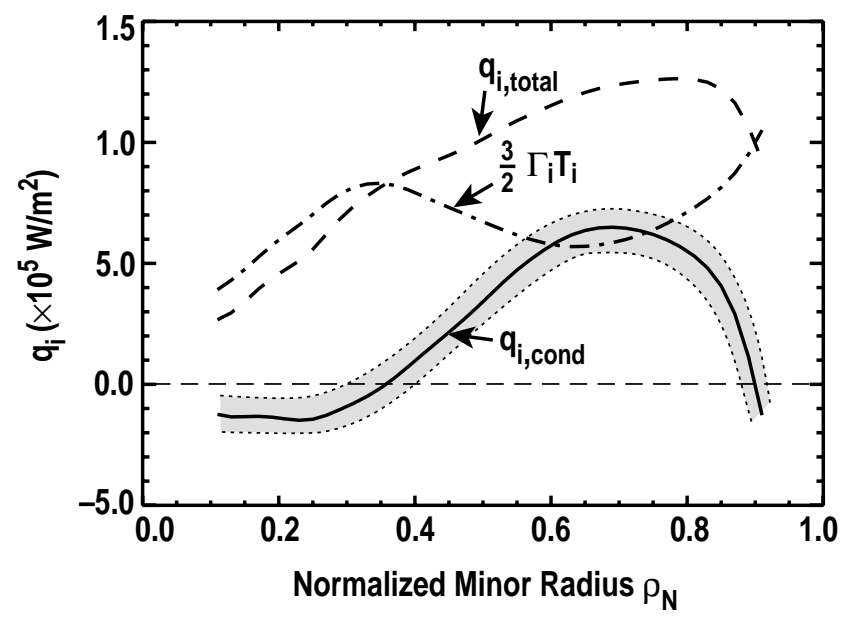

Fig. 3. Radial plots of the total ion energy flux (dashed line), the "conductive" ion heat flux $\left(q_{\text {cond }} \equiv q_{\text {total }}-3 / 2 \Gamma T\right)$ (solid line) and the 'convective' ion energy flux $\left(q_{\text {cond }} \equiv 3 / 2 \Gamma T\right)$ (dash-dot line). The appropriate errors for the conductive flux are also shown. These profiles are calculated by TRANSP for the shot shown in Fig. 1 at same time as for Fig. 2.

If $(1 / 2) \Gamma_{\mathrm{i}} T_{\mathrm{i}}$ is subtracted from $q_{\text {total }}$ instead of $(3 / 2) \Gamma_{\mathrm{i}} T_{\mathrm{i}}$, as discussed above, then the resulting value of $\chi_{i}$, defined as $\chi_{i, 1 / 2}$ is no longer negative anywhere in the plasma. Figure 4 shows a radial plot of $\chi_{i, 1 / 2}$ calculated in this way using the same $q_{\text {total }}$ as in Fig. 2. $\chi_{i, 1 / 2}$ is not significantly less than $\chi_{i, \text { neo }}$ in any region of the plasma.

The neoclassical value of $\chi_{i, n e o}$ as given by Chang-Hinton may not be valid for this discharge for $\rho<0.2$, since a basic approximation of that work, that the local radius is much larger than the poloidal Larmor radius, is not valid in this region. Also these types of plasmas have large radial electric fields in the plasma core which can affect the neoclassical orbits. Recently there has been considerable theoretical work to resolve these problems, ${ }^{28-32}$ with a wide range of results. References 29 and 30 predict that the collisional diffusion coefficient near the axis should be larger than Chang-Hinton, while Ref. 31 and 32 predict a value of a few times less and Ref. 28 predicts a value a few orders of magnitude less. This issue is still unresolved, although in no case is the value of the collisional thermal diffusion coefficient predicted to be negative. As an example, in Fig. 4 we plot the value of the neoclassical ion thermal diffusivity, reduced by the factor 
$\left[\begin{array}{c}1-\left(1-r /{ }_{\mathrm{p}}\right) \\ \left(2 q \rho_{\mathrm{L}}\right)^{L / 3} R_{0}^{1 / 3} .\end{array}\right]$, as derived in Ref. 32. $r_{\mathrm{p}}$ is 1.6 times the potato orbit width of

It should be noted that although the discharge discussed here has an internal transport barrier and relatively good ion thermal confinement, there is still significant measured fluctuations in the plasma core ${ }^{24}$ and thus, it might be expected that the thermal diffusivity is higher than neoclassical.

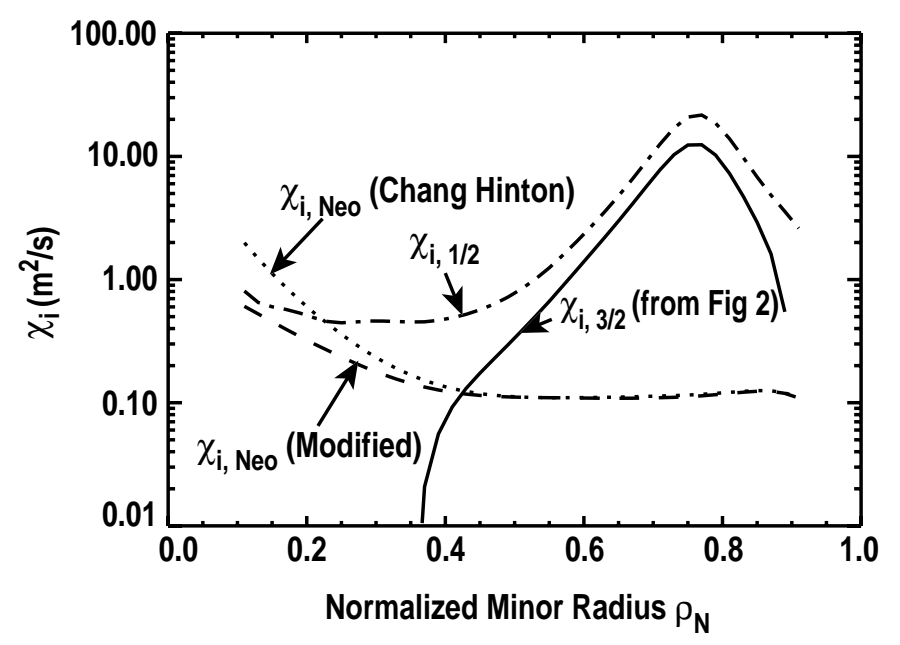

Fig. 4. Radial plot of $\chi_{i, 3 / 2}$ (solid line) at time 3.3 seconds in the discharge shown in Fig. 1. This is to be compared with Fig. 2. Also plotted is the Chang-Hinton value of the neoclassical $\chi_{i, \text { neo (dotted line). }} \chi_{\mathrm{i}, 1 / 2}$ (dash-dot line) is calculated from a "conductive" heat flux defined by $q_{\text {cond }} \equiv q_{\text {total }}-1 / 2 \Gamma T . \chi_{i, 1 / 2}$ is non-negative throughout the discharge, and is not significantly less than $\chi_{i, \text { neo throughout the discharge. Also }}$ plotted is $\lambda_{\mathrm{i}, \text { neo }} L^{1-}\left({ }^{\left.1-1 / J_{\mathrm{p}}\right)}\right]$, from Ref. 32 . 


\section{CONCLUSIONS AND SUMMARY}

One step in developing a predictive understanding of transport in a tokamak is to have a consistent way of comparing the experimentally measured transport with the theoretically predicted transport. A common way of doing this is to compare thermal or particle diffusivities. This practice probably stems from the fact that when collisional processes dominate, the diffusivities have a straight forward definition in terms of a transport matrix with specified diagonal and off diagonal terms. When turbulent transport dominates, it is still possible to develop a transport matrix, ${ }^{6-8}$ however the relative size of the diagonal and off diagonal terms depends of the type of turbulence and the off diagonal terms may completely dominate the fluxes.

For turbulent transport it is then often less instructive to compare arbitrarily defined "conductive" diffusion coefficients than to compare the measured total flux with the predicted total flux. ${ }^{15}$ This is exemplified by Eq. (27), the expression for the energy flux. There is only one term in the expression which is clearly a diffusive or "conductive" term and there is no way to unambiguously separate that term from the others since, without an exact calculation of all of the terms, the relative sizes of the terms is unknown. We are then left with the option of comparing the total fluxes or comparing effective diffusivities as defined in Eq. (28).

There is one reason to try to determine the conductive part of the energy flux. Since the definition of the conductive flux is model dependent, if the model which is used to compare to experiment gives non-physical results (such as a negative thermal diffusivity), then that model must not be correct. From the discussions presented here, it is clear that the simple model which ignores the turbulence induced stress's contribution to the fluxes must not be correct, at least in some cases. It is also clear that the inclusion of the turbulence induced stress's contribution to the fluxes removes the unphysical result of a negative thermal diffusivity and thus these effects should be considered in turbulent transport models and simulations. 


\section{References}

${ }^{1}$ A.A. Galeev and R.Z. Sagdeev, Sov.Phys. JEPT 26, 233 (1968).

${ }^{2}$ F.L. Hinton and R.D. Hazeltine, Rev. Mod. Phys. 48, 239 (1976).

${ }^{3}$ S.P. Hirschman and D.J. Sigmar, Nucl. Fusion 21, 1079 (1981).

${ }^{4}$ C.S.Chang and F.L.Hinton,Phys.Fluids 25,1493 (1982).

${ }^{5}$ See for example J.D. Callen, Phys. Fluids B 2, 2869 (1990).

6K.C. Shaing, Phys. Fluids 31, 2249 (1988).

${ }^{7}$ R. Balescu, Phys. Fluids B 2, 2100 (1990).

${ }^{8}$ H. Sugama and W. Horton, Phys. Plasmas 2, 2989 (1995).

${ }^{9}$ J.L. Luxon and L.G. Davis, Fusion Technol. 8, 441 (1985).

${ }^{10}$ R.D. Hazeltine and J.D. Meiss, "Plasma Confinement," Addison-Wesley Publishing Co., (1992).

${ }^{11}$ David Ross, Plasma Physics and Controlled Fusion 34, 137 (1992).

12P.H. Diamond and Y.-B. Kim, Phys Fluids B 3, 1626 (1991).

${ }^{13}$ T.G. Northrop, "Adiabatic Charged-Particle Motion," in Plasma Physics in Theory and Application, McGraw-Hill Book Co. (1966).

${ }^{14}$ G.R. McKee, C.C. Petty, R.E. Waltz, C.Fenzi, R.J. Fonck, et al., Nucl. Fusion 41, 1235 (2001).

15R.E. Waltz, G.M. Staebler, W. Dorland, G.W. Hammet, M. Kotchenreuther, and J.A. Konings, Phys. Plasmas 4, 2482 (1997).

16R.V. Bravenec, K.W. Gentle, B. Richards, D.W. Ross, D.C. Sing, et al., Phys. Fluids B 4, 2127 (1992).

${ }^{17}$ R.H. Rutherford, Phys. Fluids 13, 482 (1970).

${ }^{18}$ John Wesson, Tokamaks, Oxford Science Publications, $2^{\text {nd }}$ Ed. (1997).

${ }^{19}$ D.R. Baker, "Use of the Drift Kinetic Equation to Describe Electrostatic Turbulent Transport," submitted to Phys. Plasmas (2001).

${ }^{20}$ D.R. Baker, M.R. Wade, C.C. Petty, M.N. Rosenbluth, T.C. Luce, et al., Nucl. Fusion 40, 1003 (2000).

${ }^{21}$ D.F. Duchs, “3/2 or $5 / 2$ for Convective Thermal Transport?” Joint European Torus Report JET-R(89)13 (1989).

${ }^{22}$ R. Balescu, Phys. Fluids B 3, 564 (1991).

${ }^{23}$ T.E. Stringer, Plasma Phys Contr. Fusion 33, 1715 (1991).

${ }^{24}$ C.M. Greenfield, J.C. DeBoo, T.C. Luce, B.W. Stallard, E.J. Synakowski, et al., Phys. Plasmas 7, 1959 (2000).

25I.B. Bernstein and K. Molvig, Phys. Fluids 26, 1488 (1983). 
26R.J. Hawryluk, in Proc. of the Course in Physics of Plasmas Close to Thermo-nuclear Conditions, Varenna, 1979 (Commission of the European Communities, Brussels, 1980), Vol. I, p.19.

${ }^{27}$ C.M. Greenfield, K.H. Burrell, J.C. DeBoo, E.J. Doyle, B.W. Stallard, et al.,Phys. Rev. Lett. 86, 4544 (2001).

28Z. Lin, W.M. Tang and W.W. Lee, Phys. Plasmas 4, 1701 (1997).

${ }^{29}$ K.C. Shaing, R.D. Hazeltine and M.C. Zarnstorff, Phys. Plasmas 5, 771 (1997).

${ }^{30}$ K.C. Shaing and R.D. Hazeltine, Phys. Plasmas 5, 953 (1998).

${ }^{31}$ W.X. Wang, F.L. Hinton and S.K. Wong, "We need a title here," submitted to Phys. Rev. Lett. (2001).

32A. Bergmann, A.G. Peeters and S.D. Pinches, Phys. Plasmas 8, 5192 (2001). 


\section{Acknowledgments}

This is a report of work supported by the U.S. Department of Energy under Contract No. DE-AC03-99ER554463. The expert TRANSP analysis which produced the results shown in Figs. 2-3 was performed by Dr. C.M. Greenfield using data produced by the DIII-D research team. 